\title{
The Fluorescence Radiation Yield Induced in Atmosphere by Ultra-High Energy Cosmic Rays
}

\author{
M. S. A. B. Leão* \\ Centro de Ciências Naturais e Humanas, Universidade Federal do ABC, 09210-170, Santo \\ André, SP, Brazil \\ E-mail: miltonleaodufabc.edu.br
}

\section{A. Leigui de Oliveira}

Centro de Ciências Naturais e Humanas, Universidade Federal do ABC, 09210-170, Santo

André, SP, Brazil

E-mail: leiguieufabc.edu.br

\section{J. Todero Peixoto}

Centro de Ciências Naturais e Humanas, Universidade Federal do ABC, 09210-170, Santo

André, SP, Brazil

E-mail: toderocjeufabc.edu.br

\begin{abstract}
In the Extensive Air Shower (EAS) the particles deposit part or all their energy by ionization of air molecules and produce fluorescence radiation leaving a track of fluorescent light as the shower develops. A detailed study on the energy deposit of electromagnetic particles in several atmospheric layers is addressed. We take into account parameterizations for density and temperature of each layer and test two different formulations for the energy deposit in different measurements for fluorescence yield.
\end{abstract}

4th School on Cosmic Rays and Astrophysics,

August 25- September 04, 2010

Sao Paulo Brazil

* Speaker. 


\section{Introduction}

This work presents an analysis on the dependence of some calculations of the fluorescence yield (FlY) for two forms of energy deposit $d E / d x$. This is callculated using Bethe-Bloch formula [1, 2] with corrections due to the polarization effects of the medium density (parameter $\delta$ ) by Leo [3], and corrections of Sternheimer et al. for NIST (National Institute of Standards and Technology) 沟.

In the ionization, the energy loss per unit path is described, in general, by the Bethe-Bloch formula [1, 2]. In 1994, W.R.Leo [3], discribed it:

$$
-\frac{d E}{d x}=\frac{B \rho Z}{\beta^{2} A}\left[\ln \frac{\tau^{2}(\tau+2)}{2\left(\frac{I}{m_{e} c^{2}}\right)^{2}}+F(\tau)-\delta-\frac{2 C}{Z}\right]
$$

where $B=0.1535 \mathrm{MeV} /\left(\mathrm{g} / \mathrm{cm}^{2}\right), \rho$ is the density, $Z$ the atomic number, $A$ the number of mass of the absorber medium, $\tau$ the kinetic energy of incident particle in units of $m_{e} c^{2}(F(\tau)$ is a function whose form depends whether particle is a electron or positron), $\beta=v / c$ of incident particle, $I$ is the mean excitation potential, $\delta$ is the density correction (This is derived from the fact that the electric field of the particles tend to polarize the atoms on his way. Because of this polarization, electrons away from the path of the particle will be shielded and contribute less to the energy loss, and its contribution will be deducted) and $C / Z$ is a fixed for high speeds.

To compare the values of the equation 4 we used the program ESTAR [5] from NIST (National Intitute of Standards and Technology) [П]. It uses the theory of Bethe, with a density-effect correction evaluated according to Sternheimer $(1952,1982)$ [6. The data to construct the curve, showed in the figure 1., was obtained by output of this program with interval of energies between $0.001-10000 \mathrm{MeV}$.

On figure 1 there is a comparation of the $\mathrm{dE} / \mathrm{dx}$ from Leo and NIST. The results was calculated to dry air, at sea level $(760 \mathrm{mmHg}), 288^{\circ}$ Kelvin and $\rho=1,2 \mathrm{Kg} / \mathrm{m}^{3}$. The diference between the minimun $^{1}$ of both curves (about $1 \mathrm{MeV}$ ) is approximately $10 \%$.

\section{The Method}

The showers were simulated with the CORSIKA[7] program version 6617 using the hadronic model Sibyll 2.1 [8]. The chemical composition of the primaries were chosen to be proton and the energies fixed in $10^{17}, 10^{17.5}, 10^{18}, 10^{18.5}, 10^{19}, 10^{19.5}, 10^{20}$ and $10^{20.5} \mathrm{eV}$, where 1000 events were simulated for each energy. The thinning factor used was of $10^{-5}$ and the zenith angles were sorted between $0^{\circ}$ and $60^{\circ}$.

Kakimoto et al. does a parameterization of the fluorescence emission in function of the energy and altitude to the total emission of photons in the range of $300 \mathrm{~nm}$ and $430 \mathrm{~nm}$. Nagano et al. use the equation of Kakimoto et al. but with other energy normalization, where the first term is the main emission peak and the second term are the other emissions. Thus the equation for the total emission of fluorescence is given by:

$$
F l Y=\frac{\left(\frac{d E}{d x}\right)}{\left(\frac{d E}{d x}\right)_{E_{o}}} \rho\left\{\frac{A_{1}}{1+\rho B_{1} \sqrt{T}}+\frac{A_{2}}{1+\rho B_{2} \sqrt{T}}\right\}
$$

\footnotetext{
${ }^{1}$ This value is the $\mathrm{dE} / \mathrm{dx}$ of reference $(d E / d x)_{E_{o}}$.
} 


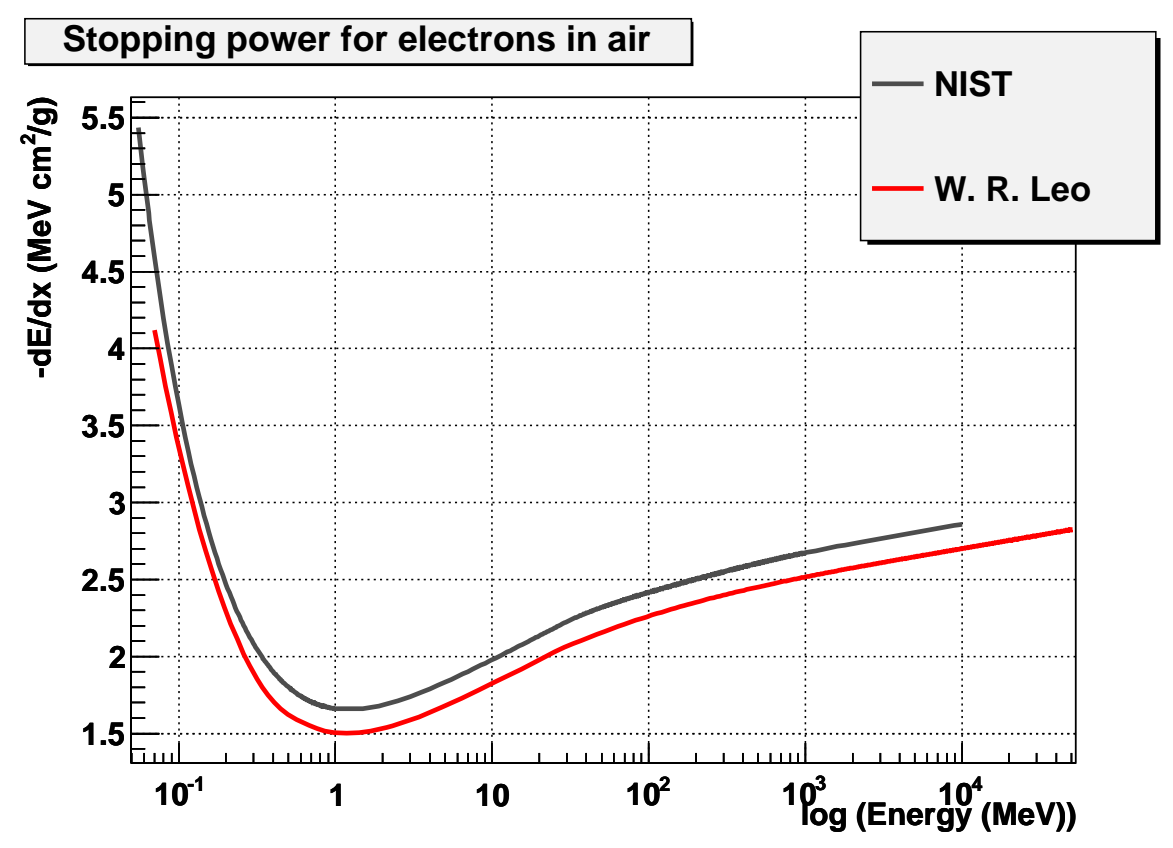

Figure 1: $\mathrm{dE} / \mathrm{dx}$ for Leo and NIST.

where $(d E / d x)_{E_{o}}$ is the energy loss normalized to $E_{o}$ (the ref. [9] take it $1.4 \mathrm{MeV}$ and the ref. [10] use $0.85 \mathrm{MeV}), \rho$ is the medium density in $\mathrm{kg} / \mathrm{m}^{3}, T$ is the medium temperature in Kelvin and the constants $A_{1}$ and $A_{2}$ in $\left[\mathrm{m}^{2} \mathrm{~kg}^{-1}\right]$, and $B_{1}$ and $B_{2}$ in $\left[\mathrm{m}^{3} \mathrm{~kg}^{-1} \mathrm{~K}^{1 / 2}\right]$ are derived from the experiments. See the values in table 1 .

\begin{tabular}{|cc|c|c|}
\hline & & $A_{1}$ & $A_{2}$ \\
\hline ref. [9] & $89.0 \pm 1.7$ & $55.0 \pm 2.2$ \\
\hline ref. [10] & $147.4 \pm 4.3$ & $69.8 \pm 12.2$ \\
\hline \hline & & $B_{1}$ & $B_{2}$ \\
\hline ref. [9] & $1.85 \pm 0.04$ & $6.50 \pm 0.33$ \\
\hline ref. [10] & $2.40 \pm 0.18$ & $20.10 \pm 6.90$ \\
\hline
\end{tabular}

Table 1: Constants used in equation (2.1).

To compare these two models with other possibility we have used the program offline. This has several models of FlY already implemented and we can be set up these different models to be used in the same data set. So, we configured this for the models from Kakimoto, Nagano, Keilhauer and Airfly. Since results are dependent of the wave-length, $\lambda$ we add all the values of FlY for each $\lambda$ in the each depth.

To make the graphics FlY with dE/dx we use showers simulated by CORSIKA, whose output data provides the number of electrons and positrons in atmospheric depth. Are 208 levels between $0-1040 \mathrm{~g} / \mathrm{cm}^{2}$, so divided in intervals of $5 \mathrm{~g} / \mathrm{cm}^{2}$. Using the formula of Kakimoto and Nagano for $\mathrm{dE} / \mathrm{dx}$ we can calculate the energy deposited for each one these levels. In the case of data table of FlY from the outputs of Offine we can not calculate the exact amount for each level, so we made 
a linear interpolation to approximate values of FIY of each level corresponding to the levels of the outputs of CORSIKA.

The version of the Offline used was v2r5p7-Godot compiled with script ape0.98.

\section{Results - Fluorescence Yield}

The influence of these corrections in $F l Y$ was higher than expected, as we can see in Fig. 2, 3. 4 and 5. The results are discrepant around 30\% near the altitude of $12 \mathrm{~km}$ between the curves Leo-Kakimoto (Offline) and NIST-Nagano (Offline).

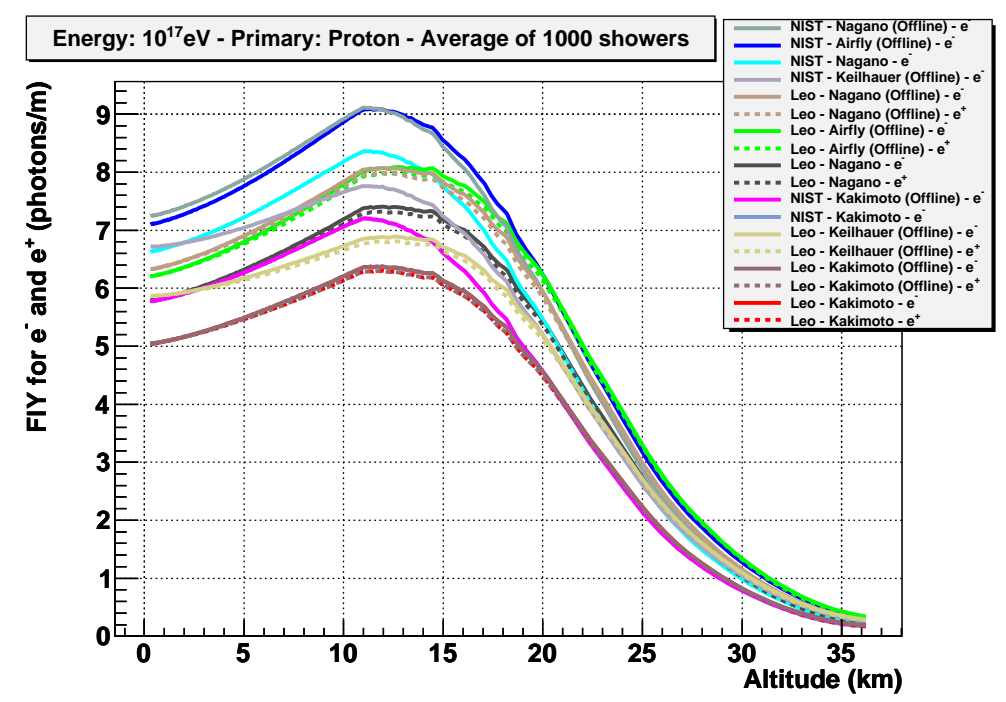

Figure 2: FlY for average of 1000 showers of proton with $10^{17} \mathrm{eV}$.

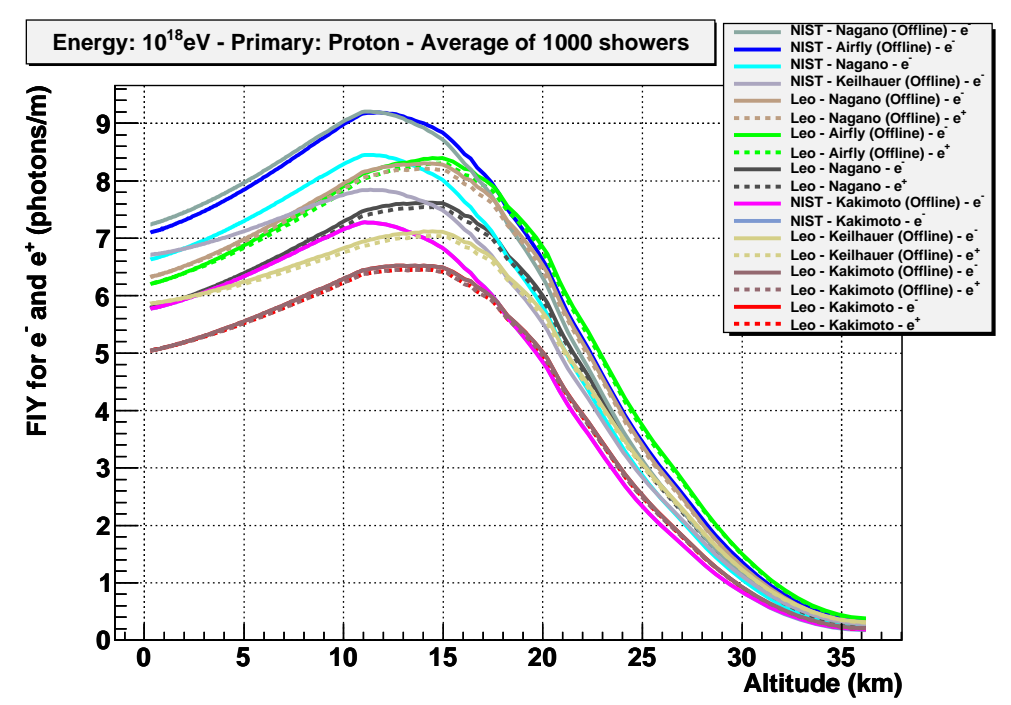

Figure 3: FlY for average of 1000 showers of proton with $10^{18} \mathrm{eV}$. 


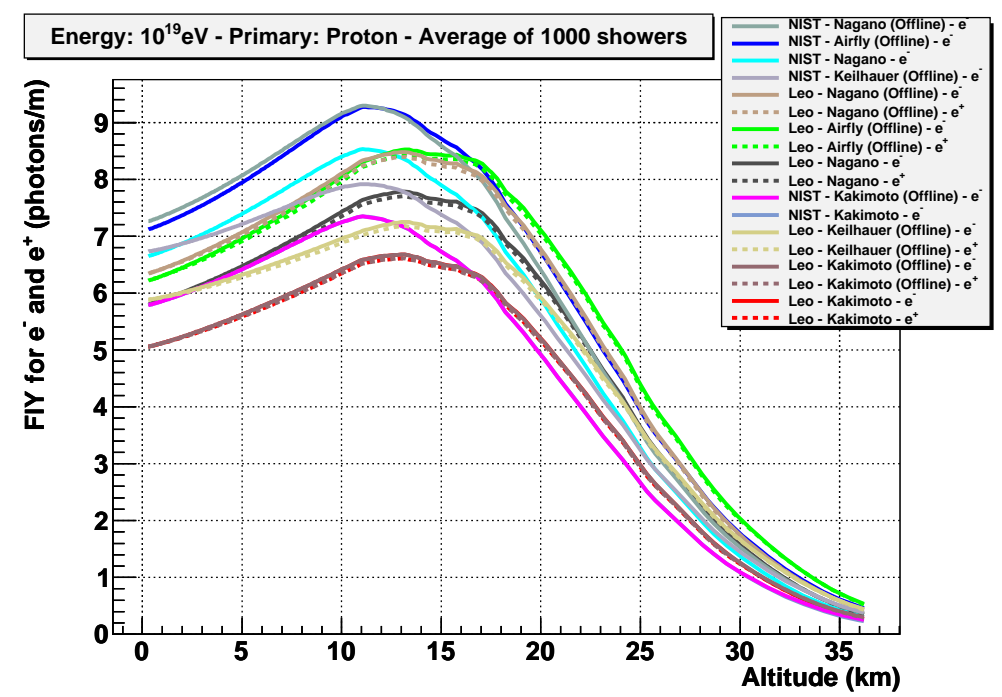

Figure 4: FlY for average of 1000 showers of proton with $10^{19} \mathrm{eV}$.

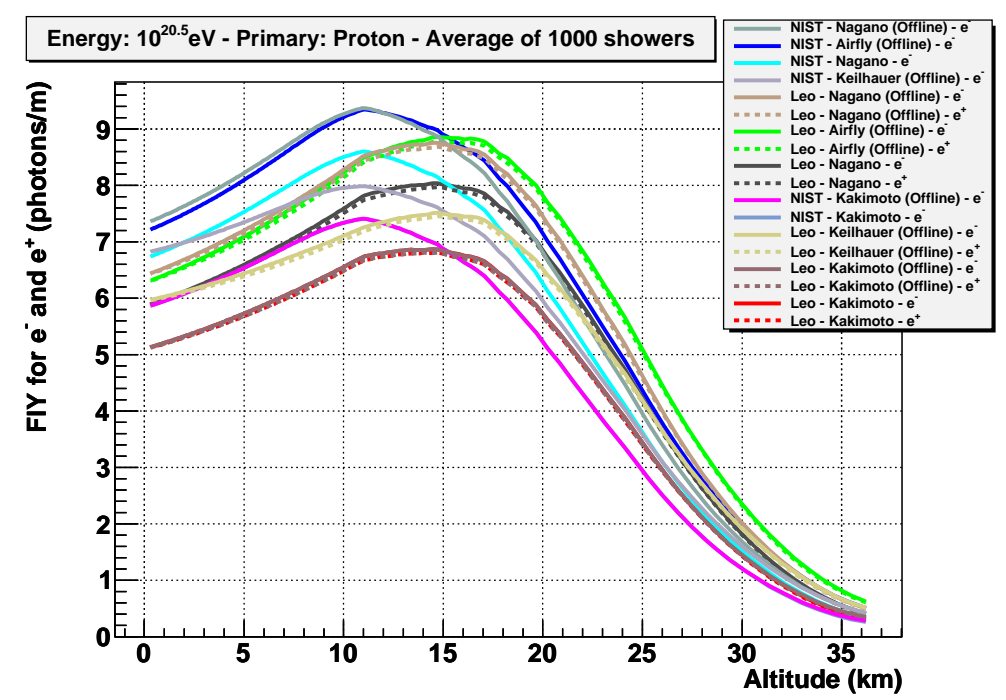

Figure 5: FlY for average of 1000 showers of proton with $10^{20,5} \mathrm{eV}$.

\section{Conclusions}

i) The combination given by equation with parameterization by Kakimoto et al. [9] is the closest to the expected results in the literature [11]; and we use this to evaluate the reliability of our results.

ii) The influence of the $\mathrm{dE} / \mathrm{dx}$ term in the $F l Y$ was much larger than expected reaching $30 \%$ between the curves Leo Kakimoto (Offline) and NIST-Nagano (Offline).

iii) The next step is to simulate showers with different chemical compositions and calculate the number of photons in the shower axis in order to check the propagation of this effect in the calculation. 


\section{Acknowledgments}

We thank the financial support given by Universidade Federal do ABC. To CENAPAD (Centro Nacional de Processamento de Alto Desempenho), NTI (Núcleo de Tecnologia e Informática UFABC) where the simulations and calculations for this work has been performed.

\section{References}

[1] H. Bethe, Ann. Phys. 5 (325) 1930.

[2] F. Bloch,Z. Phys. 81 (363) 1933.

[3] W. R. Leo, Techniques for Nuclear and Particle Physics Experiments, Springer-Verlag (37) 1994.

[4] (http://www.nist.gov/physlab/data/star/index.cfm)

[5] (http://physics.nist.gov/PhysRefData/Star/Text/ESTAR.html)

[6] R. M. Sternheimer, M. J. Berger and S. M. Seltzer, Atomic Nucl. Data Tables 30 (261) 1984

[7] D. Heck et al., CORSIKA: A Monte Carlo Code to Simulate Air Showers, Report KfK 6019, Forshungszentrum Karlsruhe 1998.

[8] R. S. Fletcher et al., Phys. Rev. D D50 (5710) 1994.

[9] K. Kakimoto, et al., Nuclear Instruments and Methods in Physics, Res.: A372 (527) 1996.

[10] M. Nagano, K. Kobayakawa, N. Sakaki and K. Ando, et al., New measurement on photon yields from air and the application to the energy estimation of primary cosmic rays, Astroparticle Physics $\mathbf{2 2}$ (235) 2004.

[11] V. de Souza, G. Medina-Tanco and J. A. Ortiz, Astropart. Phys. 25 (129) (2006). 